\title{
Recent Clinical Trials on Natural Products and Traditional Chinese Medicine Combating the COVID-19
}

\author{
Shivraj Hariram Nile $^{1} \cdot$ Guoyin Kai $^{1}$ (B)
}

Received: 13 December 2020/Accepted: 17 December 2020/Published online: 30 December 2020

(C) Association of Microbiologists of India 2020

\begin{abstract}
The novel severe acute respiratory syndrome coronavirus 2 (SARS-CoV-2) causing potentially fatal coronavirus disease-19 (COVID-19), with a significant health and economic burden around the globe. Currently many clinical studies are undergoing but still there is no any specific approved therapy or drug established for effective treatment of COVID-19. This review aimed to analyses various clinical studies which have been registered in www.clinicaltrials.gov and http://www.chictr.org. cn were registered with natural plant-based medicines and Traditional Chinese medicine (TCM) for discovering effective treatment and prevention of COVID-19. Total 46 and 64 natural drug and TCM interventions were identified which mainly determined the preventive strategies and possible treatments for COVID-19 infection. We identified that most of the clinical trial undergoing on natural compound like heparin and vitamin $\mathrm{C}$ as therapeutic agents and immune boosters for against COVID-19. Traditional Chinese medicines and herbal medicines can be effectively used as a preventive therapy against COVID-19 and after successful clinical trials and these potential therapies can be promoted by countries around the world.
\end{abstract}

Keywords COVID-19 - Natural products - TCM - SARS$\mathrm{CoV}-2 \cdot$ Clinical trials

Supplementary Information The online version contains supplementary material available at (https://doi.org/10.1007/s12088020-00919-x)

Guoyin Kai

guoyinkai1@126.com

1 Laboratory of Medicinal Plant Biotechnology, College of Pharmacy, Zhejiang Chinese Medical University, Hangzhou 310053, Zhejiang, China

\section{Introduction}

In December 2019, Wuhan City of Hubei Province, China first reported the emergence of a novel coronavirus outbreak named "Severe Acute Respiratory Syndrome Coronavirus 2" (SARS-CoV-2), the cause of the 2019 Coronavirus Disease (COVID-19) a pandemic threat to global health [1]. According to the World Health Organization (WHO) the detailed information provided on the global spread of COVID-19 around the world as pandemic, as of December 13, 2020, the outbreak had spread to more than 215 countries, with $>72,645,205$ confirmed cases and $>1,616,908$ confirmed deaths cases [2]. SARS-CoV-2 makes entry in host cell through the attachment of S protein (spike proteins) to ACE2 (angiotensin-converting enzyme 2) that is mediated via host cell-produced serine protease TMPRSS211 (transmembrane serine protease 211) [3]. Search for possible potential drug candidates based on their target location on virion and host cells, mainly targeting key proteins and their mechanisms of spread; such as inhibiting ACE2 and TMPRS211 proteins, producing polyclonal antibodies against viral S protein by blocking its binding, and inhibiting virus replication inside the host cell [4]. Infectious diseases are caused by microbial pathogens transmitted by various animals, and found that they had certain degree of resistance towards some antiviral drugs and antibiotics. Genomics is important in a diverse biotechnological application, including the developing antipathogenic drugs and therapies [5-7]. Unfortunately, to date, there are no any approved potential drugs or vaccine candidates have been granted for the prevention of COVID-19. The significant increase in the number of progressive cases of confirmed COVID-19 patients indicates that there is an urgent need to develop a potential and preventive acute anti-COVID medicine or therapy, thus an 
anti-COVID-19 potential drug development is the research process for the invention and establishment of preventive vaccines or therapeutic drugs that reduce the severity of COVID-19 pandemic as early as possible [8]. Around the globe several national and international research scientists, institutions and pharma companies are working collaboratively on therapeutic drug and vaccine development, also studies are in pipelined with various stages of preclinical or clinical research [9]. In March 2020, the WHO launched the "SOLIDARITY Trial" to register thousands of people infected with the COVID-19 to evaluate the use of existing potential antiviral compounds and therapies; mainly remdesivir, chloroquine, hydroxychloroquine, ritonavir/ lopinavir, and interferon- $\beta$ which showing most promising therapeutic effects against SARS-CoV-2 infection to prevent COVID-19 infection among humans [10, 11]. Therapeutic drug and vaccine development are a multistep process and typically requires many years to assure safety and efficacy against viral diseases, as a conservative estimate of time needed to prove a safe, effective therapy or vaccine, therefore, some national and international regulatory agencies such as the European Medical Agency (EMA) and the Food and Drug Administration (FDA) approved procedures to speed up clinical trials. [12]. Currently the drugs which are previously used to as antiviral, antimalarial, and anti-HIV agents are currently being evaluated against prevention and treatment of COVID-19 infection [13]. Plant-based natural herbal formulations and compounds are widely used as rich resource for development of novel antiviral drugs against various viruses including coronavirus, influenza viruses, human immunodeficiency virus, herpes simplex virus, severe acute respiratory syndrome (SARS) virus, Middle East respiratory syndrome (MERS) virus, and hepatitis $\mathrm{B}$ and $\mathrm{C}$ viruses [14]. To date, number of Chinese herbs and numerous naturally occurring bioactive compounds have been reported in revealing the antiviral effect on the influence of the viral life cycle and replication (Fig. 1). Number of natural compounds were screened against viral proteins using in silico methods. The possible target proteins are main protease (3CLpro, also named 3-chymotrypsin-like protease), helicase, papain like protease (also named as PLpro), RNA-dependent RNA polymerase called as RdRp, and viral spike protein [15].

\section{Current Status of Drug Development}

\section{Natural Products (Herbal Medicines)}

As the long-durability of antibiotics developed by various pathogenic microorganisms is a newly emerging problem, it has developed to control the counterattack of other microbial pathogens. Therefore, vaccines have limited efficacy as a therapeutic target for the treatment of various bacterial and viral infections [16-19]. Scientists and researchers around the world have made tremendous efforts to develop preventive therapeutic drugs and vaccines against COVID-19. Preclinical studies have demonstrated the potential of multiple responses towards SARS-CoV-2 infection, but large-scale trials are still needed for proper management and control of COVID-19 spread and development as community spread. Therefore, there is an urgent need to develop potentially effective therapies to treat, prevent and improve mortality from severe COVID-19 $[2,8]$. However, development of drugs and vaccines are a multi-step, time-consuming and expensive systematic process, with a high natural attrition rate. In the current global emergency, the normal speed of drug development is not acceptable. As a result, there has been a strong interest in the reuse of existing antiviral drugs, which have previously been widely used to treat viral infections such as influenza, Severe Acute Respiratory Syndrome Coronavirus (SARS-CoV), human immunodeficiency virus (HIV), the Middle East Respiratory Syndrome Coronavirus (MERS-CoV), hepatitis B (HBV), hepatitis C (HCV), and other pathological antiviral plant based natural and chemically synthesized drugs [9, 13].

\section{Traditional Chinese Medicine (TCM)}

Currently various studies and reports are available on use of TCM products to treat respiratory tract infections for COVID-19 treatment and preventions [20]. In addition, through clinical practice and Western medicine research, TCM is used as the third version of the comprehensive treatment plan, showing the promising role of China's successful battle against COVID-19 [21]. The products from TCM like ShuFeng JieDu and Lianhua Qingwen capsules shown to exert effective influenza antiviral effects and also showed very promising synergistic antiviral effects with use of Western medicine products [22]. The patented TCM medicines were recommended aaccording to the Chinse national guidance provided on treatment of COVID-19 under medical observation period including the effective supplementation of Shufengjiedu capsule and Jinhuaqinggan granules. These formulations prepared using various plant extracts through TCM were found to be related to multiple signaling pathways involved during the treatment of COVID-19 developed due to SARS-CoV-2 infection. Shufengjiedu capsule mainly targeting ERK pathway, modulating anti-inflammatory and immunomodulation activity, while Jinhuaqinggan granules targeting TNF, MAPK and T cell receptor signaling pathways, and Lianhuaqingwen an TCM formulation with broad-spectrum antiviral effect and effective to treat influenza virus's 


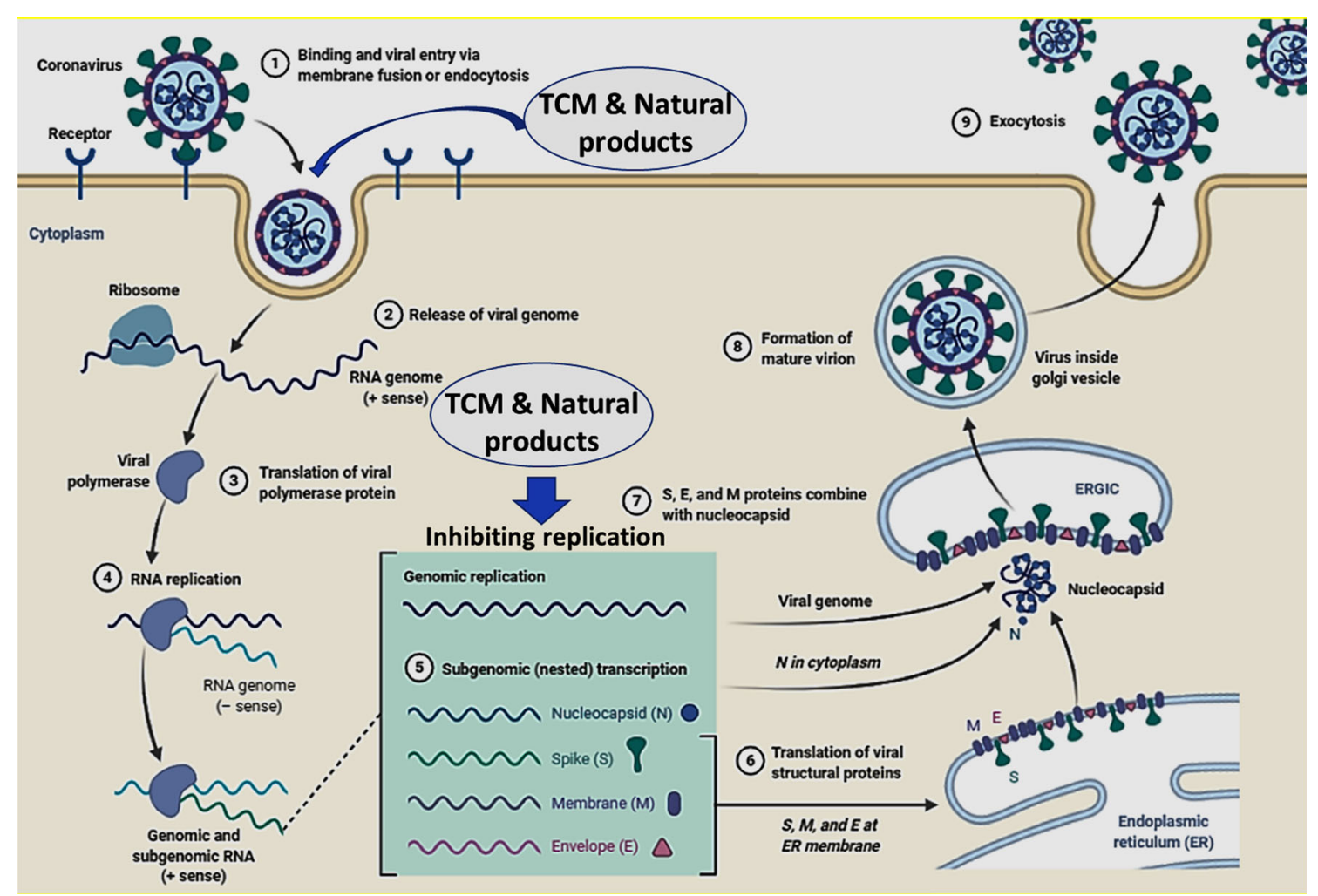

Fig. 1 SARS-CoV-2 virus replication and possible effects of TCM and natural products currently under clinical trials (Table S1 and S2)

infection, also inhibited significantly the replication of SARS-CoV-2 with reduced pro-inflammatory cytokines production [23]. According to the details and guidelines provided for use of TCM protocols, there are basically five types of traditional Chinese medicine. Huoxiang Zhengqi Wan (Agastache Qi-correcting pills), Fangfeng Tongsheng Zhiji (Saposhnikoviae heat removal preparation), Lianhua Qingwen Zhiji (Forsythiae and Honeysuckle flower insect removal preparations), and Shufeng Jiedu Keli (Wind-expelling and toxin removal granules). In addition to the use of these the traditional medicine preparations, the TCM program also recommends the use of four other Chinese medicine injections for COVID-19 treatment, including Xuebijing injections, Shengmai injections, Shenfu injections and Xiyanping injections [24]. Recently it was found that the Liu Shen capsule, have been proven to have a wide spectrum antiviral and immunomodulatory effects and also known to have effective treatment against SARSCoV-2 [25], some of these currently being studied for clinical trials which were discussed in Table S1. The current research data showed that various TCM products and their combinations had an effective treatment options for COVID-19. The synergistic effect of TCM with Western medicine also used as an effective treatment for COVID-19 [22]. However, there are few published research studies ongoing and underway in clinical trials on TCM and other
Chinese medicinal products for the treatment of COVID19.

\section{Study Design}

In this article, we conduct the systematic review on different natural and plant based bioactive compounds undergoing clinical trials for COVID-19 treatment and prevention. Here, we evaluated different natural dugs recently being used in randomized controlled trials (RCTs) for pandemic COVID-19. Herein, we searched current clinical trials on natural products as potential therapy for COVID-19 using www.clinicaltrials.gov and http://www. chictr.org.cn to identify clinical trials for "COVID19", SARS-Cov-2", Coronavirus, "Natural compounds", "Herbal", "Plant", "Phenolics", "Flavonoids", "Hormones", "Carotenoids", "Traditional Chinese Medicine (TCM)", "Chinese Drugs", and "Vitamins" (Fig. 2). All the interventional trials were included during this study. We excluded treatment and prevention trials those are not having the term "COVID19" and "SARS-CoV-2" in the study title. In this study we independently performed the data extraction and analysis in triplicates. The methods for review preformed and described Cochrane Handbook [20] and methods previously elsewhere [9]. 


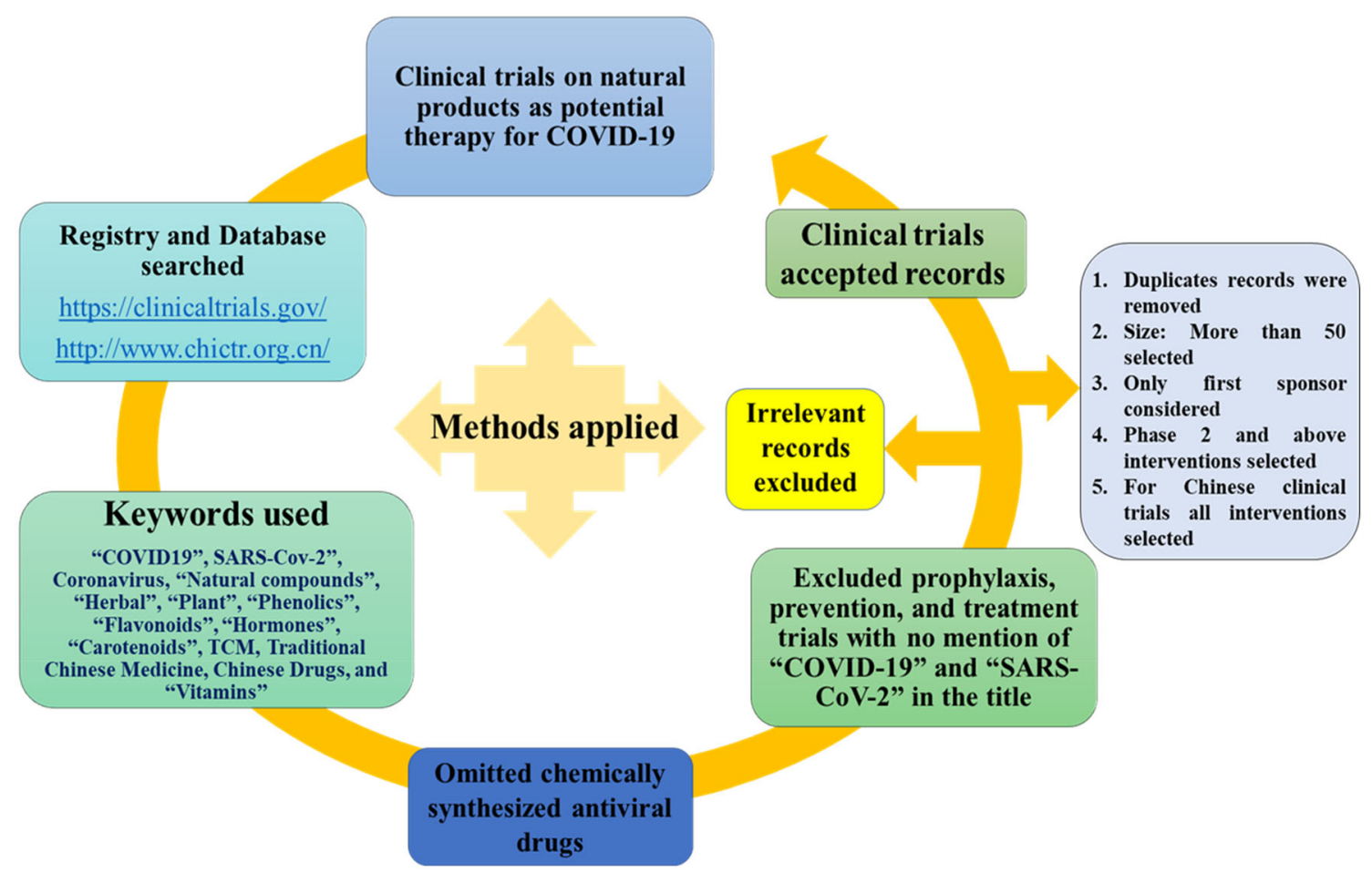

Fig. 2 Flow chart for methods used for selection of therapies in clinical trials

\section{Discussion}

In the context of the current COVID-19 outbreak, we conducted a systematic review on recent clinical investigations through online registration, analyzed the current clinical trials under going on COVID-19 therapies mainly includes natural products and Traditional Chinese Medicines, as well as other plant based potential therapeutic agents used against prevention of COVID-19 by searching through www.clinicaltrials.gov and http://www. chictr.org.cn, resulted were presented in Table S1 and S2. This will help to give recent insights on current investigational advances on natural products as potential targets with practical guidance for potential future strategies. Total 46 natural drug interventions were identified which mainly targeted on both the treatment of patients and preventative strategies to control COVID-19 infection. In this systematic review we found that most of the clinical trials are undergoing on heparin and vitamin $\mathrm{C}$ as therapeutic natural agents and TCM preparations, as an immune booster during prevention and treatment of COVID-19. Heparin an FDA-approved anticoagulant agent which prevents blood clots caused by certain medical conditions, which may neutralize SARS-CoV-2, causative viral agent of COVID19. The virus SARS-CoV-2 attaches to host human cell by using its spike protein to ACE2 protein and causing infection to human cells [26]. However, it was found that the heparin binds tightly to viral $\mathrm{S}$ protein, blocking the attachment to ACE2 protein of human cells, it potentially helps to prevent SARS-CoV-2 infection from developing COVID-19. Heparin, thus considered to possess non-anticoagulant effects which might be helpful in management of COVID-19 through direct antiviral entry and providing anti-inflammatory mechanism in human cells [26, 27]. Vitamin C (Ascorbic acid) acts as strong antioxidant, antiinflammatory and immunomodulator having beneficial healthy effects in severe and critical illness to human health. Vitamin C triggers the human vascular integrity and cellular immunity by serving as cofactor during generation of endogenous catecholamines. Humans may need more vitamin $\mathrm{C}$ during unhealthy condition or infection due to formation oxidative stress, severe microbial attack and sepsis in body. In COVID-19, acute respiratory distress syndrome (ARDS) and sepsis have developed easily due to viral infection in human cells, so vitamin $\mathrm{C}$ supplemented with medications can reduce inflammation and vascular damage in patients having COVID-19 [28, 29]. Thus, only phase 3 and size more than 300 clinical trials were noted in this study for these two drugs. We also found that extensive studies and research were important for analysis of these studied natural bioactive compounds as potential therapies against COVID-19 regardless of lack of scientific basis, insufficient information on use of these bio-active plant based natural compounds and limited applicability to mainstream medical practice. 
Recently, there has been no specific approval for the treatment or prevention of COVID-19 drugs, vaccines or therapies from FDA and WHO, so many researchers and scientists are committed to reusing possible antiviral drugs as potential therapies for COVID-19. To overcome from this challenging time of pandemic, the researchers and scientist performing clinical trials on various synthetic, natural and TCM antimicrobial therapies on COVID-19 in a safe, compliant, and ethnical manner. Currently intensive clinical trials of COVID-19 using various drugs are ongoing around the world and China. However, the factors such as small sample size, poor drug quality, and long completion time, many researchers are unable to obtain reliable and high-quality clinical evidence for COVID-19 treatment. In order to effectively respond to current health emergencies, further exploration is needed to determine the best strategies to change the research behavior of against this COVID-19 pandemic study [30]. The limitations of this work are to include a smaller sample size of studies and limited only to natural products and TCM as possible and potential therapeutic agents for COVID-19 through online registries under clinical investigation from http:// www.chictr.org and www.clinicaltrials.gov. Given the evolving nature of recent drug development against COVID-19, there are many more studies must be needed and made available through proper clinical trials on TCM and natural drugs that can strongly studied to design potential therapies and data points to compare further developments in the coming months for possible control of COVID-19 pandemic.

\section{Conclusion}

The systematic assessment provided in this study identified a total of 46 and 64 natural plant-based medicines and traditional Chinese medicine interventions, respectively, focusing on prevention strategies and the treatment of SARS-CoV-2 infection cased COVID-19 in patients around the globe. The results show that natural compounds (such as heparin and vitamin C) are effective natural products and traditional Chinese medicine-based therapies for combating the COVID-19 and immune boosters. However, all analytical studies have brought major risks to bias and have major methodological flaws. Therefore, there is still a lack of clinical evidence to support the therapeutic use of these natural accruing heparin and vitamin C. At present, some randomized clinical trials on natural products and Chinese medicine are under way, with more rigorous research design and more participants. However, due to factors such as small sample size, poor drug quality and long completion time it's difficult to find the reliable and high-quality clinical evidence on COVID-19 treatments.
Acknowledgements This work was supported by National Natural Science Fund (81522049, 31571735, 31270007), Zhejiang Natural Science Fund (LY20H280008), Zhejiang Provincial Program for the Cultivation of High-level Innovative Health talents.

\section{References}

1. Zhao W, Zhang J, Meadows ME, Liu Y, Hua T, Fu B (2020) A systematic approach is needed to contain COVID-19 globally. Sci Bull 65(11):876-878. https://doi.org/10.1016/j.scib.2020.03.024

2. WHO Coronavirus Disease (COVID-19) Dashboard. World Health Organisation (WHO). 2020; https://covid19.who.int/

3. Patel SKS, Lee JK, Kalia VC (2020) Deploying biomolecules as antii-COVID-19 agents. Indian J Microbiol 60:263-268. https:// doi.org/10.1007/s12088-020-00893-4

4. Rishi P, Thakur K, Vij S, Rishi L, Singh A, Kaur IP, Patel SKS, Lee JK, Kalia VC (2020) Diet, gut microbiota and COVID-19. Indian J Microbiol 60:420-429. https://doi.org/10.1007/s12088020-00908-0

5. Kalia VC, Raju SC, Purohit HJ (2011) Genomic analysis reveals versatile organisms for quorum quenching enzymes: acyl-homoserine lactone-acylase and-lactonase. Open Microbiol J 5:1-11. https://doi.org/10.2174/1874285801105010001

6. Kalia VC, Wood TK, Kumar P (2014) Evolution of resistance to quorum-sensing inhibitors. Microb Ecol 68:13-23. https://doi. org/10.1007/s00248-013-0316-y

7. Kumar P, Patel SKS, Lee JK, Kalia VC (2013) Extending the limits of Bacillus for novel biotechnological applications. Biotechnol Adv 31:1543-1561. https://doi.org/10.1016/j.bio techadv.2013.08.007

8. Lythgoe MP, Middleton P (2020) Ongoing clinical trials for the management of the COVID-19 pandemic. Trends Pharmacol Sci 41(6):363-382. https://doi.org/10.1016/j.tips.2020.03.006

9. Desai A, Kulkarni A, Rajkumar SV, Gyawali B (2020) Clinical trial endpoints in severe COVID-19. Mayo Clin Proc 95(8):1578-1580. https://doi.org/10.1016/j.mayocp.2020.05.025

10. Kupferschmidt K, Cohen J (2020) WHO launches global megatrial of the four most promising coronavirus treatments? Sci Mag. https://doi.org/10.1126/science.abb8497

11. Nile SH, Nile A, Qiu J, Li L, Jia X, Kai G (2020) COVID-19: pathogenesis, cytokine storm and therapeutic potential of interferons. Cytokine Growth Factor Rev 53:66-70. https://doi.org/10. 1016/j.cytogfr.2020.05.002

12. Joppi R, Bertele V, Vannini T, Garattini S, Banzi R (2020) Food and drug administration vs European medicines agency: review times and clinical evidence on novel drugs at the time of approval. Br J Clin Pharmacol 86:170-174. https://doi.org/10. 1111/bcp. 14130

13. Shereen MA, Khan S, Kazmi A, Bashir N, Siddique R (2020) COVID-19 infection: origin, transmission, and characteristics of human coronaviruses. J Adv Res 24:91-98. https://doi.org/10. 1016/j.jare.2020.03.005

14. Xian Y, Zhang J, Bian Z, Zhou H, Zhang Z, Lin Z, Xu H (2020) Bioactive natural compounds against human coronaviruses: a review and perspective. Acta Pharma Sin B 10(7):1163-1174. https://doi.org/10.1016/j.apsb.2020.06.002

15. Mani JS, Johnson JB, Steel JC, Broszczak DA, Neilsen PM, Walsh KB, Naiker M (2020) Natural product-derived phytochemicals as potential agents against coronaviruses: a review. Virus Res 284:197989. https://doi.org/10.1016/j.virusres.2020. 197989 
16. Kalia VC (2013) Quorum sensing inhibitors: an overview. Biotechnol Adv 31:224-245. https://doi.org/10.1016/j.bio techadv.2012.10.004

17. Kalia VC, Patel SKS, Kang YC, Lee J-K (2019) Quorum sensing inhibitors as antipathogens: biotechnological applications. Biotechnol Adv 37:68-90. https://doi.org/10.1016/j.biotechadv. 2018.11.006

18. Kalia VC, Purohit HJ (2011) Quenching the quorum sensing system: potential antibacterial drug targets. Crit Rev Microbiol 37:121-140. https://doi.org/10.3109/1040841X.2010.532479

19. Parasuraman P, Devadatha B, Sarma VV, Ranganathan S, Ampasala DR, Reddy D, Kumavath R, Kim I-W, Patel SKS, Kalia VC, Lee J-K, Siddhardha B (2020) Inhibition of microbial quorum sensing mediated virulence factors by Pestalotiopsis sydowiana. J Microbiol Biotechnol 30:571-582. https://doi.org/ 10.4014/jmb.1907.07030

20. Cumpston M, Li T, Page MJ, Chandler J, Welch VA, Higgins JP, Thomas J (2019) Updated guidance for trusted systematic reviews: a new edition of the Cochrane handbook for systematic reviews of interventions. Cochrane Database Syst Rev 10:ED000142

21. Ren JL, Zhang AH, Wang XJ (2020) Traditional Chinese medicine for COVID-19 treatment. Pharmacol Res 155:104743. https://doi.org/10.1016/j.phrs.2020.104743

22. Qing GC, Zhang H, Bai Y, Luo Y (2020) Traditional Chinese and Western medicines jointly beat COVID-19 pandemic. Chin J Integr Med 6:403-404. https://doi.org/10.1007/s11655-020-30956

23. Runfeng L, Yunlong H, Jicheng H, Weiqi P, Qinhai M, Yongxia $S$ et al (2020) Lianhuaqingwen exerts anti-viral and antiinflammatory activity against novel coronavirus (SARS-CoV-2). Pharmacol Res 156:104761. https://doi.org/10.1016/j.phrs.2020. 104761

24. Liu M, Gao Y, Yuan Y, Yang K, Shi S, Zhang J, Tian J (2020) Efficacy and safety of integrated traditional Chinese and western medicine for corona virus disease 2019 (COVID-19): a systematic review and meta-analysis. Pharmacol Res 158:104896. https://doi.org/10.1016/j.phrs.2020.104896

25. Ma QH, Pan WQ, Li RF, Liu B, Li CF, Xie YQ et al (2020) Liu Shen capsule shows antiviral and anti-inflammatory abilities against novel coronavirus SARS-CoV-2 via suppression of NFkB signaling pathway. Pharmacol Res 158:104850. https://doi. org/10.1016/j.phrs.2020.104850

26. Kim SY, Jin W, Sood A, Montgomery DW, Grant OC, Fuster MM, Fu L, Dordick JS, Woods RJ, Zhang F, Linhardt RJ (2020) Characterization of heparin and severe acute respiratory syndrome-related coronavirus 2 (SARS-CoV-2) spike glycoprotein binding interactions. Antiviral Res 181:104873. https://doi.org/ 10.1016/j.antiviral.2020.104873

27. Hippensteel JA, Lariviere WB, Colbert JF, Langouët-Astrié CJ, Schmidt EP (2020) Heparin as a therapy for COVID-19: current evidence and future possibilities. Am J Physiol Lung Cell Mol Physiol 319(2):L211-L217. https://doi.org/10.1152/ajplung. 00199.2020

28. Wei XB, Wang ZH, Liao XL, Guo WX, Wen JY, Qin TH, Wang SH (2020) Efficacy of vitamin in patients with sepsis: an updated meta-analysis. Eur J Pharmacol 868:172889. https://doi.org/10. 1016/j.ejphar.2019.172889

29. Boretti A, Banik BK (2020) Intravenous vitamin C for reduction of cytokines storm in acute respiratory distress syndrome. PharmaNutrition 12:100190. https://doi.org/10.1016/j.phanu.2020. 100190

30. Yu L (2020) Restoring good health in elderly with diverse gut microbiome and food intake restriction to combat COVID-19. Ind J Microbiol. https://doi.org/10.1007/s12088-020-00913-3

Publisher's Note Springer Nature remains neutral with regard to jurisdictional claims in published maps and institutional affiliations. 